\title{
DIFFERENCE EQUATIONS IN WEIGHTED SPACES OF SEQUENCES
}

\author{
NADIR V. IbAdov, IL'DAR Kh. Musin
}

\begin{abstract}
Let $\varphi=\left\{\varphi_{m}\right\}_{m=1}^{\infty}$ be a family of convex functions $\varphi_{m}$ on $\mathbb{R}^{n}$ with certain growth conditions. With a help of restrictions of functions $\varphi_{m}$ on $\mathbb{Z}^{n}$ a weighted space of functions on $\mathbb{Z}^{n}$ denoted as $A_{\varphi}$ is defined. Linear continuous functionals on this space in terms of their FourierLaplace transform are described. This description and functional analysis methods allowed to study surjectivity of difference operators on $A_{\varphi}$ and spectral synthesis problem in the kernel of such operators for a special case of a family $\varphi$.
\end{abstract}

Keywords: sequence spaces, linear difference equation, entire functions, duality.

\section{Introduction}

Let $\varphi=\left\{\varphi_{m}\right\}_{m=1}^{\infty}$ be a family of convex functions $\varphi_{m}: \mathbb{R}^{n} \rightarrow \mathbb{R}$ such that:

1) $\lim _{x \rightarrow \infty} \frac{\varphi_{m}(x)}{\|x\|}=+\infty$ for each $m \in \mathbb{N}\left(\|\cdot\|\right.$ is the Euclidean norm on $\left.\mathbb{R}^{n}\right)$;

2) $\exists A>0 \forall m \in \mathbb{N} \exists B_{m} \geqslant 0$ :

$$
\varphi_{m}(x)-\varphi_{m+1}(x) \geqslant A \ln (1+\|x\|)-B_{m}, \quad x \in \mathbb{R}^{n} .
$$

For each $m \in \mathbb{N}$ let

$$
A\left(\varphi_{m}\right)=\left\{f: \mathbb{Z}^{n} \rightarrow \mathbb{C} \text { such that } p_{m}(f)=\sup _{\alpha \in \mathbb{Z}^{n}} \frac{|f(\alpha)|}{e^{\varphi_{m}(\alpha)}}<\infty\right\} .
$$

Obviously, for each $m \in \mathbb{N} A\left(\varphi_{m+1}\right) \subset A\left(\varphi_{m}\right)$. Let $A_{\varphi}=\bigcap_{m=1}^{\infty} A\left(\varphi_{m}\right)$. Thus, for $n=1$ elements of $A_{\varphi}$ are two-sided sequences, for $n>1$ elements of $A_{\varphi}$ are multiple sequences. For brevity elements of $A_{\varphi}$ will be simply called sequences. Sometimes we denote a sequence $f$ as $(f(\alpha))_{\alpha \in \mathbb{Z}^{n}}$.

Under usual operations of addition and multiplication by complex numbers $A_{\varphi}$ is a linear space. Endow $A_{\varphi}$ with the topology of projective limit of the spaces $A\left(\varphi_{m}\right)$. Obviously, $A_{\varphi}$ is a separable Fréchet space.

The research of the second author was supported by grants from RFBR (11-01-00572, 1101-97019, 12-01-97004).

2010 Mathematics Subject Classification: primary: 39A10; secondary: 47B39, 40B05 
In this article a description of the strong dual space of $A_{\varphi}$ in terms of FourierLaplace transform of linear continuous functionals on $A_{\varphi}$ as some space of periodic entire functions in $\mathbb{C}^{n}$ is obtained. Such a description allowed to study surjectivity of difference operators on $A_{\varphi}$ and spectral synthesis problem in the kernel of such operators for a special case of a family $\varphi$.

Note that Fourier-Laplace transform of linear continuous functionals on sequence spaces was succesfully applied by many authors to study various analysis problems in these spaces. For example, L.A. Rubel and B.A. Taylor [6] considered spaces of all two-sided and one-sided sequences of complex numbers of at most exponential growth and proved some "polynomial" approximation theorems in these spaces by dualizing a gap theorem of C. Rényi [4] for periodic entire functions. This approach was also applied by A.A. Borichev [1] to describe the solutions of convolution equations in certain spaces of two-sided and one-sided sequences of exponential growth.

We shall use the following notations. For $u=\left(u_{1}, \ldots, u_{n}\right) \in \mathbb{R}^{n}\left(\mathbb{C}^{n}\right), v=$ $\left(v_{1}, \ldots, v_{n}\right) \in \mathbb{R}^{n}\left(\mathbb{C}^{n}\right)\langle u, v\rangle=u_{1} v_{1}+\cdots+u_{n} v_{n}$ and $\|u\|$ denotes the Euclidean norm in $\mathbb{R}^{n}\left(\mathbb{C}^{n}\right)$.

For $\alpha=\left(\alpha_{1}, \ldots, \alpha_{n}\right) \in \mathbb{Z}^{n}, x=\left(x_{1}, \ldots, x_{n}\right) \in \mathbb{R}^{n}, z=\left(z_{1}, \ldots, z_{n}\right) \in \mathbb{C}^{n}$ $|\alpha|=\alpha_{1}+\ldots+\alpha_{n}, \alpha^{\prime}=\left(\alpha_{2}, \ldots, \alpha_{n}\right), x^{\alpha}=x_{1}^{\alpha_{1}} \cdots x_{n}^{\alpha_{n}}, z^{\alpha}=z_{1}^{\alpha_{1}} \cdots z_{n}^{\alpha_{n}}, D^{\alpha}=$ $\frac{\partial^{|\alpha|}}{\partial z_{1}^{\alpha_{1}} \cdots \partial z_{n}^{\alpha_{n}}}$.

For multi-indices $\alpha=\left(\alpha_{1}, \ldots, \alpha_{n}\right), \beta=\left(\beta_{1}, \ldots, \beta_{n}\right) \in \mathbb{Z}_{+}^{n}$ the notation $\beta \leqslant \alpha$ indicates that $\beta_{j} \leqslant \alpha_{j}(j=1,2, \ldots, n)$.

For multi-indices $\alpha=\left(\alpha_{1}, \ldots, \alpha_{n}\right), \beta=\left(\beta_{1}, \ldots, \beta_{n}\right) \in \mathbb{Z}_{+}^{n}$ such that $\beta \leqslant \alpha$ let $C_{\alpha}^{\beta}=\prod_{j=1}^{n} C_{\alpha_{j}}^{\beta_{j}}$ where $C_{\alpha_{j}}^{\beta_{j}}$ are the combinatorial numbers.

For $r>0$ and $z \in \mathbb{C}^{n}$ let $B(z, r)=\left\{\zeta \in \mathbb{C}^{n}:\|\zeta-z\|<r\right\}$.

For a locally convex space $X$ let $X^{\prime}$ be the space of linear continuous functionals on $X$ and let $X^{*}$ be the strong dual space.

For a function $\Phi \in C\left(\mathbb{R}^{n}\right)$ such that $\lim _{x \rightarrow \infty} \frac{\Phi(x)}{\|x\|}=+\infty$ let

$$
\begin{array}{ll}
\Phi^{\star}(x):=\sup _{\alpha \in \mathbb{Z}^{n}}(\langle x, \alpha\rangle-\Phi(\alpha)), & x \in \mathbb{R}^{n} ; \\
\Phi^{*}(x):=\sup _{y \in \mathbb{R}^{n}}(\langle x, y\rangle-\Phi(y)), & x \in \mathbb{R}^{n} .
\end{array}
$$

Recall that $\Phi^{*}$ is called the Young conjugate of the function $\Phi$. It is well known that if $\Phi$ is convex on $\mathbb{R}^{n}$ then $\left(\Phi^{*}\right)^{*}=\Phi$.

Obviously, for each $z \in \mathbb{C}^{n}$ the sequence $f_{z}: \alpha \in \mathbb{Z}^{n} \rightarrow e^{-i\langle z, \alpha\rangle}$ belongs to $A_{\varphi}$ since for each $m \in \mathbb{N}$

$$
p_{m}\left(f_{z}\right)=\sup _{\alpha \in \mathbb{Z}^{n}} \frac{\left|e^{-i\langle z, \alpha\rangle}\right|}{e^{\varphi_{m}(\alpha)}}=\exp \left(\sup _{\alpha \in \mathbb{Z}^{n}}\left(\langle\operatorname{Im} z, \alpha\rangle-\varphi_{m}(\alpha)\right)\right)=e^{\varphi_{m}^{\star}(\operatorname{Imz})}<\infty .
$$

Thus, for each linear continuous functional $S$ on $A_{\varphi}$ the function $\hat{S}(z)=S\left(f_{z}\right)$ is correctly defined on $\mathbb{C}^{n}$. It is called the Fourier-Laplace transform of $S$. The mapping $\mathcal{F}: S \in A_{\varphi}^{*} \rightarrow \hat{S}$ is called the Fourier-Laplace transformation. 
For each $m \in \mathbb{N}$ let

$$
\begin{aligned}
P\left(\varphi_{m}^{\star}\right)=\left\{F \in H\left(\mathbb{C}^{n}\right): F(z+2 \pi l)=F(z) \text { for all } z \in \mathbb{C}^{n}, l \in \mathbb{Z}^{n}\right. \\
\text { and such that } \left.\|F\|_{m}=\sup _{z \in \mathbb{C}^{n}} \frac{|F(z)|}{e^{\varphi_{m}^{\star}(\text { Imz })}}<\infty\right\} .
\end{aligned}
$$

Let $\varphi^{\star}=\left\{\varphi_{m}^{\star}\right\}_{m=1}^{\infty}$ and $P_{\varphi^{\star}}=\bigcup_{m=1}^{\infty} P\left(\varphi_{m}^{\star}\right)$. Under usual operations of addition and multiplication by complex numbers $P_{\varphi^{\star}}$ is a linear space. Endow $P_{\varphi^{\star}}$ with the topology of inductive limit of the spaces $P\left(\varphi_{m}^{\star}\right)$.

The main results of the paper are the following.

Theorem 1.1. The mapping $\mathcal{F}: S \in A_{\varphi}^{*} \rightarrow \hat{S}$ establishes an isomorphism between the spaces $A_{\varphi}^{*}$ and $P_{\varphi^{\star}}$.

Theorem 1.1 is proved in the second section. In the third section we apply Theorem 1.1 to study difference operators in $A_{\varphi}$. For $f \in A_{\varphi}$ and $h \in \mathbb{Z}^{n}$ define a sequence $f_{h}$ by the rule: $f_{h}(\alpha)=f(\alpha+h), \alpha \in \mathbb{Z}^{n}$. Let $H$ be a finite subset of $\mathbb{Z}^{n}$ and for $h \in H$ let $\gamma_{h}$ be a complex number.

Theorem 1.2. Let $\varphi$ satisfies the following additional conditions:

$\left.\mathrm{i}_{1}\right)$ for each $m \in \mathbb{N}$ there exist numbers $a_{m}>0, b_{m}>0$ and $\mu_{m}>1$ such that

$$
\varphi_{m}(x) \geqslant a_{m}\|x\|^{\mu_{m}}-b_{m}, \quad x \in \mathbb{R}^{n} ;
$$

$\left.\mathrm{i}_{2}\right)$ for each $m \in \mathbb{N}$ there exists $d_{m}>0$ such that for all $x \in \mathbb{R}^{n}$ and $\xi=$ $\left(\xi_{1}, \ldots, \xi_{n}\right) \in \mathbb{R}^{n}$ with $\left|\xi_{j}\right| \leqslant 1(j=1, \ldots, n)$

$$
\varphi_{m+1}(x+\xi) \leqslant \varphi_{m}(x)+d_{m} .
$$

Then the equation $\sum_{h \in H} \gamma_{h} f_{h}=g$ is solvable in $A_{\varphi}$ for each $g \in A_{\varphi}$.

For $\mu \in \mathbb{Z}_{+}^{n}$ and $\zeta \in \mathbb{C}^{n}$ define a sequence $E_{\mu, \zeta}$ by the rule: $\alpha \in \mathbb{Z}^{n} \rightarrow$ $\alpha^{\mu} e^{-i\langle\alpha, \zeta\rangle}$. Note that in view of the second condition on $\varphi E_{\mu, \zeta}$ is in $A_{\varphi}$.

Denote the zero element of $A_{\varphi}$ by $\mathbf{0}$. Let $W$ be the set of all solutions $f \in A_{\varphi}$ of the equation $\sum_{h \in H} \gamma_{h} f_{h}=\mathbf{0}$ and $\mathcal{E}$ be the set of all solutions of the form $E_{\mu, \zeta}$.

Theorem 1.3. Let the family $\varphi$ satisfies the conditions of Theorem 1.2 and $\mathcal{E}$ is not empty. Then a closure of a linear envelope of $\mathcal{E}$ in $A_{\varphi}$ is $W$.

\section{Space $A_{\varphi}$ and its dual}

To note some special properties of the spaces $A_{\varphi}$ and $P_{\varphi^{\star}}$ we need to remember definitions of $\left(M^{*}\right)$-space and $\left(L N^{*}\right)$-space from [7], [8].

$\left(M^{*}\right)$-space is a locally convex space $F$ which is the projective limit of a sequence of normed spaces $F_{k}$ with linear continuous mappings $g_{m k}: F_{k} \rightarrow F_{m}$, $m<k$, such that $g_{k, k+1}$ is compact for each $k \in \mathbb{N}$. 
$\left(L N^{*}\right)$-space is a locally convex space $E$ which is the inductive limit of an increasing sequence of normed spaces $E_{k}$ such that the unit ball of $E_{k}$ is relatively compact in $E_{k+1}$ for each $k \in \mathbb{N}$, i.e. such that the inclusion map from $E_{k}$ into $E_{k+1}$ is compact.

It is known that if $E$ (the inductive limit of an increasing sequence of normed spaces $\left.E_{k}\right)$ is an $\left(L N^{*}\right)$-space then a set $B$ is bounded in $E$ iff for some $m \in \mathbb{N}$ it is contained in $E_{m}$ and bounded there ([7], Theorem 1).

It is easy to show that the inclusions $I_{m}: A\left(\varphi_{m+1}\right) \rightarrow A\left(\varphi_{m}\right)$ are compact for each $m \in \mathbb{N}$. So $A_{\varphi}$ is an $\left(M^{*}\right)$-space. Therefore, $A_{\varphi}$ is a reflexive space ([7], Proposition 7).

From conditions on $\varphi$ it follows that $\lim _{x \rightarrow \infty}\left(\varphi_{m+1}^{\star}(x)-\varphi_{m}^{\star}(x)\right)=+\infty$. Using this fact and Montel's theorem it can be shown that the mappings $J_{m}: P\left(\varphi_{m}^{\star}\right) \rightarrow$ $P\left(\varphi_{m+1}^{\star}\right)$ are compact for each $m \in \mathbb{N}$. Thus, the space $P_{\varphi^{\star}}$ is an $\left(L N^{*}\right)$-space.

Lemma 2.1. For each $S \in A_{\varphi}^{\prime}$ we have $\hat{S} \in P_{\varphi^{\star}}$.

Proof. First show that for $S \in A_{\varphi}^{\prime} \hat{S}$ is an entire function. For $f \in A_{\varphi}$ and $N \in \mathbb{N}$ define the mapping $f_{N}: \mathbb{Z}^{n} \rightarrow \mathbb{C}$ by the rule: $f_{N}(\alpha)=f(\alpha)$ for $|\alpha| \leqslant N$, $f_{N}(\alpha)=0$ for $|\alpha|>N$. Then for each $s \in \mathbb{N}$

$$
p_{s}\left(f-f_{N}\right)=\sup _{|\alpha|>N} \frac{|f(\alpha)|}{e^{\varphi_{s}(\alpha)}} \leqslant p_{s+1}(f) \exp \left(\sup _{|\alpha|>N}\left(\varphi_{s+1}(\alpha)-\varphi_{s}(\alpha)\right)\right) .
$$

Taking into account condition 2) on $\varphi$ we conclude that $p_{s}\left(f-f_{N}\right) \rightarrow 0$ as $N \rightarrow \infty$. This means that $f_{N} \rightarrow f$ in $A_{\varphi}$ as $N \rightarrow \infty$. Hence, $S\left(f_{N}\right) \rightarrow S(f)$ as $N \rightarrow \infty$. For each $\alpha \in \mathbb{Z}^{n}$ let $e_{\alpha}$ be the mapping $e_{\alpha}: \mathbb{Z}^{n} \rightarrow \mathbb{C}$ acting by the rule: $e_{\alpha}(\beta)=1$ if $\alpha=\beta, e_{\alpha}(\beta)=0$ if $\alpha \neq \beta$. Then $f_{N}=\sum_{|\alpha| \leqslant N} f(\alpha) e_{\alpha}$. Consequently, $S(f)=\lim _{n \rightarrow \infty} S\left(f_{N}\right)=\sum_{\alpha \in \mathbb{Z}^{n}} \gamma(\alpha) f(\alpha)$, where $\gamma(\alpha)=S\left(e_{\alpha}\right)$. In particular,

$$
\hat{S}(z)=\sum_{\alpha \in \mathbb{Z}^{n}} \gamma(\alpha) e^{-i\langle z, \alpha\rangle}, \quad z \in \mathbb{C}^{n} .
$$

Obviously, $\hat{S}(z+2 \pi l)=\hat{S}(z)$ for all $z \in \mathbb{C}^{n}$ and $l \in \mathbb{Z}^{n}$. Since $S$ is a linear continuous functional on $A_{\varphi}$ then for some $m \in \mathbb{N}$ and $c>0$

$$
|S(f)| \leqslant c p_{m}(f), \quad f \in A_{\varphi} .
$$

From this we have for each $\alpha \in \mathbb{Z}^{n}$

$$
|\gamma(\alpha)|=\left|S\left(e_{\alpha}\right)\right| \leqslant c p_{m}\left(e_{\alpha}\right)=c e^{-\varphi_{m}(\alpha)} .
$$

Using the second condition on $\varphi$ we can choose $k \in \mathbb{N}$ so that the inequality $\sum_{\alpha \in \mathbb{Z}^{n}} e^{\varphi_{m+k}(\alpha)-\varphi_{m}(\alpha)}<\infty$ holds. Now using (3) we have for each $z \in \mathbb{C}^{n}$

$$
\begin{aligned}
|\hat{S}(z)| & =\left|\sum_{\alpha \in \mathbb{Z}^{n}} \gamma(\alpha) e^{-i\langle z, \alpha\rangle}\right| \leqslant c \sum_{\alpha \in \mathbb{Z}^{n}} e^{-\varphi_{m}(\alpha)+\langle\alpha, \operatorname{Im} z\rangle} \\
& \leqslant c e^{\varphi_{m+k}^{\star}(\operatorname{Im} z)} \sum_{\alpha \in \mathbb{Z}^{n}} e^{\varphi_{m+k}(\alpha)-\varphi_{m}(\alpha)} .
\end{aligned}
$$


From this it follows that the series $\sum_{\alpha \in \mathbb{Z}^{n}} \gamma(\alpha) e^{-i\langle z, \alpha\rangle}$ converges uniformly on compact subsets of $\mathbb{C}^{n}$. Hence, $\hat{S}$ is an entire function and $\hat{S}$ is in $P_{\varphi^{\star}}$.

Remark 2.1. Using the representation (2), inequality (3) and the second condition on $\varphi$ it is easy to see that for each $S \in A_{\varphi}^{\prime}$ we have

$$
\left.\left(D^{\nu} \hat{S}\right)(z)=S\left((-i \alpha)^{\nu} e^{-i\langle z, \alpha\rangle}\right)_{\alpha \in \mathbb{Z}^{n}}\right), \quad \nu \in \mathbb{Z}_{+}^{n}, z \in \mathbb{C}^{n} .
$$

Proof of Theorem 1.1. By Lemma 2.1 $\mathcal{F}(S) \in P_{\varphi^{\star}}$ for each $S \in A_{\varphi}^{*}$.

Let us show now that the linear mapping $\mathcal{F}$ is continuous. But first note that the topology of $A_{\varphi}^{*}$ can be described as follows. For each $k \in \mathbb{N}$ let $W_{k}=\{f \in$ $\left.A_{\varphi}: p_{k}(f) \leqslant 1\right\}$ and $W_{k}^{0}=\left\{S \in A_{\varphi}^{\prime}:|S(f)| \leqslant 1, \forall f \in W_{k}\right\}$ be a polar of $W_{k}$ in $A_{\varphi}^{\prime}$. Let $T_{k}=\bigcup_{\alpha>0}\left(\alpha W_{k}^{0}\right)$ be a vector subspace in $A_{\varphi}^{\prime}$ generated by $W_{k}^{0}(k \in \mathbb{N})$. Define a topology in $T_{k}$ with a help of the norm

$$
N_{k}(S)=\sup _{f \in W_{k}}|S(f)|, \quad S \in T_{k}
$$

Obviously, $A_{\varphi}^{\prime}=\bigcup_{k=1}^{\infty} T_{k}$. Define in $A_{\varphi}^{\prime}$ the topology $\lambda$ of an inductive limit of spaces $T_{k}$. Since $A_{\varphi}$ is a reflexive space then the strong topology in $A_{\varphi}^{\prime}$ coincides with the topology $\lambda\left([2]\right.$, chapter 8). Now let $S \in T_{k}, k \in \mathbb{N}$. Then $|S(f)| \leqslant$ $N_{k}(S), f \in W_{k}$. Hence, $|S(f)| \leqslant N_{k}(S) p_{k}(f), f \in A_{\varphi}$. Putting here $f=f_{z}$ with $z \in \mathbb{C}^{n}$ and using (1) we obtain that

$$
|\hat{S}(z)| \leqslant N_{k}(S) e^{\varphi_{k}^{\star}(\operatorname{Im} z)} .
$$

From this it follows that $\|\hat{S}\|_{k} \leqslant N_{k}(S), \quad S \in T_{k}(k=1,2, \ldots)$. Thus, $\mathcal{F}$ is continuous.

Let us prove that $L$ is injective. Let $S \in A_{\varphi}^{*}$ and $\hat{S}(z)=0$ for each $z \in \mathbb{C}^{n}$. For some $m \in \mathbb{N}$ and $c>0$ we have $|S(f)| \leqslant c p_{m}(f), f \in A_{\varphi}$. As it was shown in the proof of Lemma 2.1 the functional $S$ admits the representation

$$
S(f)=\sum_{\alpha \in \mathbb{Z}^{n}} \gamma(\alpha) f(\alpha), \quad f \in A_{\varphi},
$$

where complex numbers $\gamma(\alpha)$ satisfy the inequality

$$
|\gamma(\alpha)| \leqslant c e^{-\varphi_{m}(\alpha)}, \quad \alpha \in \mathbb{Z}^{n} .
$$

From this representation we have for each $x \in \mathbb{R}^{n}$

$$
\hat{S}(x)=\sum_{\alpha \in \mathbb{Z}^{n}} \gamma(\alpha) e^{-i\langle x, \alpha\rangle}=0
$$

Therefore, coefficients $\gamma(\alpha)=0$ for all $\alpha \in \mathbb{Z}^{n}$ and $S$ is a zero functional. Thus, $\mathcal{F}$ is injective. 
Now we prove that $\mathcal{F}$ is surjective. Let $F \in P_{\varphi^{\star}}$. Then $F \in P\left(\varphi_{m}^{\star}\right)$ for some $m \in \mathbb{N}$. Represent $F(x)$ by the Fourier series

$$
F(x)=\sum_{\alpha \in \mathbb{Z}^{n}} c_{\alpha} e^{-i\langle x, \alpha\rangle}, \quad x \in \mathbb{R}^{n} .
$$

For each $\alpha \in \mathbb{Z}^{n}$ we have

$$
c_{\alpha}=\frac{1}{(2 \pi)^{n}} \int_{-\pi}^{\pi} \cdots \int_{-\pi}^{\pi} F(x) e^{i\langle x, \alpha\rangle} d x .
$$

In view of periodicity of $F$ for each $\alpha \in \mathbb{Z}^{n}$ and $y \in \mathbb{R}^{n}$

$$
c_{\alpha}=\frac{1}{(2 \pi)^{n}} \int_{-\pi}^{\pi} \cdots \int_{-\pi}^{\pi} F(x+i y) e^{i\langle x+i y, \alpha\rangle} d x .
$$

From this we get

$$
\left|c_{\alpha}\right| \leqslant \frac{\|F\|_{m}}{(2 \pi)^{n}} \int_{-\pi}^{\pi} \cdots \int_{-\pi}^{\pi} e^{\varphi_{m}^{\star}(y)-\langle y, \alpha\rangle} d x, \quad \alpha \in \mathbb{Z}^{n}, y \in \mathbb{R}^{n} .
$$

Since $\varphi_{m}^{\star}(y) \leqslant \varphi_{m}^{*}(y)$ for every $y \in \mathbb{R}^{n}$ then for each $\alpha \in \mathbb{Z}^{n}$ we have

$$
\left|c_{\alpha}\right| \leqslant\|F\|_{m} \exp \left(\inf _{y \in \mathbb{R}^{n}}\left(\left(\varphi_{m}^{*}(y)-\langle y, \alpha\rangle\right)\right)=\|F\|_{m} e^{-\left(\varphi_{m}^{*}\right)^{*}(\alpha)}=\|F\|_{m} e^{-\varphi_{m}(\alpha)} .\right.
$$

Define a functional $S$ on $A_{\varphi}$ by the formula $S(f)=\sum_{\alpha \in \mathbb{Z}^{n}} c_{\alpha} f(\alpha), f \in A_{\varphi}$. Using the estimate (4) and the second condition on $\varphi$ it is easy to see that the linear functional $S$ is continuous. Obviously, $\hat{S}(z)=F(z), z \in \mathbb{C}^{n}$. Thus, $\mathcal{F}$ is surjective.

By the open mapping theorem [2], [5] $\mathcal{F}^{-1}$ is continuous. Thus, $\mathcal{F}$ is a topological isomorphism and the proof of theorem is complete.

\section{A difference operator on $\boldsymbol{A}_{\varphi}$}

3.1. In the proof of Theorems 1.2 and 1.3 we will use Lemma 3.2. But first let us prove the following auxiliary result.

Lemma 3.1. Let a function $h: \mathbb{Z}^{n} \rightarrow \mathbb{R}$ be such that for some constants $\nu>1$, $C>0$ and $D>0$

$$
h(\alpha) \geqslant C\|\alpha\|^{\nu}-D, \quad \alpha \in \mathbb{Z}^{n} .
$$

Then there exists a constant $M_{h}>0$ such that

$$
\left|h^{\star}(\xi)-h^{\star}(x)\right| \leqslant M_{h}
$$

for all $x, \xi \in \mathbb{R}^{n}$ satisfying the condition $\|\xi-x\| \leqslant(1+\|x\|)^{-\frac{1}{\nu-1}}$. 
Proof. For each $x \in \mathbb{R}^{n}$ let $\alpha(x) \in \mathbb{Z}^{n}$ be a point where the supremum of the function $u_{x}: \alpha \in \mathbb{Z}^{n} \rightarrow\langle x, \alpha\rangle-h(\alpha)$ over $\mathbb{Z}^{n}$ is attained.

First prove that there exists a constant $m_{h}>0$ not depending on $x$ such that

$$
\|\alpha(x)\| \leqslant m_{h} \cdot\left(1+\|x\|^{\frac{1}{\nu-1}}\right) .
$$

Using (5) we have for each $\alpha \in \mathbb{Z}^{n}$

$$
u_{x}(\alpha) \leqslant\|\alpha\| \cdot\|x\|-C\|\alpha\|^{\nu}+D .
$$

Since $h^{\star}(x)=\sup _{\alpha \in \mathbb{Z}^{n}} u_{x}(\alpha) \geqslant-h(0)$ (here 0 is the zero element of $\mathbb{Z}^{n}$ ) then the supremum of $u_{x}$ over $\mathbb{Z}^{n}$ is attained on the set

$$
G_{x}=\left\{\alpha \in \mathbb{Z}^{n}:\|\alpha\| \cdot\|x\| \geqslant C\|\alpha\|^{\nu}-D-h(0)\right\} .
$$

Put $L_{h}=D+h(0)$. By the condition on $h$ we have $L_{h} \geqslant 0$. For each $\lambda \geqslant 0$ denote by $T_{\lambda}$ the set of solutions of the inequality

$$
\lambda t \geqslant C t^{\nu}-L_{h},
$$

belonging to $\mathbb{R}_{+}$. This set is a segment of a form $\left[0, t_{\lambda}\right]$, where $t_{\lambda}<\infty$. Let us estimate $t_{\lambda}$ from above. We have $\lambda t_{\lambda}=C t_{\lambda}^{\nu}-L_{h}$. If $t_{\lambda} \geqslant 1$ then

$$
\lambda=C t_{\lambda}^{\nu-1}-\frac{L_{h}}{t_{\lambda}} \geqslant C t_{\lambda}^{\nu-1}-L_{h}
$$

From this $t_{\lambda} \leqslant\left(\frac{\lambda+L_{h}}{C}\right)^{\frac{1}{\nu-1}}$. Taking into account a case $t_{\lambda} \in[0,1)$ we have

$$
t_{\lambda} \leqslant\left(\frac{\lambda+L_{h}}{C}\right)^{\frac{1}{\nu-1}}+1 .
$$

From this if $0 \leqslant \lambda \leqslant 1$ then $t_{\lambda} \leqslant\left(\frac{1+L_{h}}{C}\right)^{\frac{1}{\nu-1}}+1$. Moreover if $\lambda>1$ then $t_{\lambda} \leqslant \lambda^{\frac{1}{\nu-1}}\left(\frac{1+L_{h}}{C}\right)^{\frac{1}{\nu-1}}+1$. Let $m_{h}:=\left(\frac{1+L_{h}}{C}\right)^{\frac{1}{\nu-1}}+1$. Then

$$
t_{\lambda} \leqslant m_{h}\left(1+\lambda^{\frac{1}{\nu-1}}\right) \text {. }
$$

Let $d_{\lambda}:=m_{h}\left(1+\lambda^{\frac{1}{\nu-1}}\right)$. Then $T_{\lambda} \subseteq\left[0, d_{\lambda}\right]$. Since $\alpha \in G_{x} \Leftrightarrow\|\alpha\| \in T_{\|x\|}$, then for all $\alpha \in G_{x}$ we have

$$
\|\alpha\| \leqslant m_{h} \cdot\|x\|^{\frac{1}{\nu-1}}+m_{h} .
$$

In particular,

$$
\|\alpha(x)\| \leqslant m_{h} \cdot\|x\|^{\frac{1}{\nu-1}}+m_{h} .
$$

Further, for all $x, \xi \in \mathbb{R}^{n}$ such that $\|\xi-x\| \leqslant(1+\|x\|)^{\frac{1}{1-\nu}}$ we have

$$
\begin{aligned}
h^{\star}(\xi)-h^{\star}(x) & =\sup _{\alpha \in \mathbb{Z}^{n}}(\langle\xi, \alpha\rangle-h(\alpha))-\sup _{\alpha \in \mathbb{Z}^{n}}(\langle x, \alpha\rangle-h(\alpha)) \\
& \leqslant(\langle\xi, \alpha(\xi)\rangle-h(\alpha(\xi)))-(\langle x, \alpha(\xi)\rangle-h(\alpha(\xi))) \\
& =\langle\xi-x, \alpha(\xi)\rangle \leqslant\|\xi-x\|\|\alpha(\xi)\| \leqslant(1+\|x\|)^{\frac{1}{1-\nu}} m_{h}\left(1+\|\xi\|^{\frac{1}{\nu-1}}\right) \\
& \leqslant \frac{2 m_{h}(1+\|x\|)^{\frac{1}{\nu-1}}}{(1+\|x\|)^{\frac{1}{\nu-1}}}=2 m_{h} .
\end{aligned}
$$


Similarly,

$$
\begin{aligned}
h^{\star}(x)-h^{\star}(\xi) & \leqslant\langle x-\xi, \alpha(x)\rangle \leqslant\|x-\xi\|\|\alpha(x)\| \\
& \leqslant \frac{m_{h}\left(1+\|x\| \frac{1}{\nu-1}\right)}{(1+\|x\|)^{\frac{1}{\nu-1}}} \leqslant \frac{2 m_{h}(1+\|x\|)^{\frac{1}{\nu-1}}}{(1+\|x\|)^{\frac{1}{\nu-1}}}=2 m_{h} .
\end{aligned}
$$

From these estimates we get (6) with $M_{h}=2 m_{h}$.

Lemma 3.2. Let the family $\varphi$ satisfies the conditions $\left.\mathrm{i}_{1}\right)$ and $\mathrm{i}_{2}$ ) of Theorem 1.2. Then for each $m \in \mathbb{N}$ :

there exists a constant $K_{m}>0$ such that

$$
\left|\varphi_{m}^{\star}(\xi)-\varphi_{m}^{\star}(x)\right| \leqslant K_{m}
$$

for all $x, \xi \in \mathbb{R}^{n}$ satisfying the condition $\|\xi-x\| \leqslant(1+\|x\|)^{-\frac{1}{\mu_{m}-1}}$;

$$
\varphi_{m+1}^{\star}(x)-\varphi_{m}^{\star}(x) \geqslant\|x\|-d_{m}, \quad x \in \mathbb{R}^{n} .
$$

Proof. The inequality (7) holds in view of Lemma 3.1. So let us prove the inequality (8). For each $x \in \mathbb{R}^{n}$ and $m \in \mathbb{N}$ let $\alpha_{m}(x) \in \mathbb{Z}^{n}$ be a point where the supremum of the function $u_{x}: \alpha \in \mathbb{Z}^{n} \rightarrow\langle x, \alpha\rangle-\varphi_{m}(\alpha)$ over $\mathbb{Z}^{n}$ is attained. Let $\theta(x)$ be the point in $\mathbb{R}^{n}$ with coordinates $\theta_{j}$ defined as follows: $\theta_{j}=\frac{x_{j}}{\left|x_{j}\right|}$ if $x_{j} \neq 0$ and $\theta_{j}=0$ if $x_{j}=0(j=1, \ldots, n)$. Then using the condition $\left.\mathrm{i}_{2}\right)$ we have

$$
\begin{aligned}
\varphi_{m+1}^{\star}(x)-\varphi_{m}^{\star}(x) & \geqslant\langle x, \theta(x)\rangle-\varphi_{m+1}\left(\alpha_{m}(x)+\theta(x)\right)+\varphi_{m}\left(\alpha_{m}(x)\right) \\
& \geqslant\left|x_{1}\right|+\cdots+\left|x_{n}\right|-d_{m} \geqslant\|x\|-d_{m} .
\end{aligned}
$$

Thus, the inequality (8) is proved.

Remark 3.1. For each $m \in \mathbb{N}$ and $x \in \mathbb{R}^{n}$ let $y_{m}(x)$ be a point where the supremum of the function $v_{x}: y \in \mathbb{R}^{n} \rightarrow\langle x, y\rangle-\varphi_{m}(y)$ over $\mathbb{R}^{n}$ is attained. Let $\alpha \in \mathbb{Z}^{n}$ be the nearest point to $y_{m}(x)$. Then

$$
\varphi_{m}^{*}(x)=\left\langle x, y_{m}(x)\right\rangle-\langle x, \alpha\rangle+\langle x, \alpha\rangle-\varphi_{m+1}(\alpha)+\varphi_{m+1}(\alpha)-\varphi_{m}\left(y_{m}(x)\right) .
$$

From this representation using the condition $\mathrm{i}_{2}$ ) we have

$$
\varphi_{m}^{*}(x) \leqslant n\|x\|+\varphi_{m+1}^{\star}(x)+d_{m} .
$$

Now by (8) we get for some $d_{m, n}>0$

$$
\varphi_{m}^{*}(x) \leqslant \varphi_{m+n+1}^{\star}(x)+d_{m, n}, \quad x \in \mathbb{R}^{n} .
$$

On the other hand, for each $m \in \mathbb{N}$ and $x \in \mathbb{R}^{n} \quad \varphi_{m}^{\star}(x) \leqslant \varphi_{m}^{*}(x)$.

Two last inequalities mean that the space $P_{\varphi^{\star}}$ coincides with the space $P_{\varphi^{*}}$ which is an inductive limit of the spaces

$$
\begin{gathered}
P\left(\varphi_{m}^{*}\right)=\left\{F \in H\left(\mathbb{C}^{n}\right): F(z+2 \pi l)=F(z) \text { for all } z \in \mathbb{C}^{n}, l \in \mathbb{Z}^{n}\right. \\
\text { and such that } \left.\|F\|_{m}=\sup _{z \in \mathbb{C}^{n}} \frac{|F(z)|}{e^{\varphi_{m}^{*}(\operatorname{Im} z)}}<\infty\right\} .
\end{gathered}
$$

Here $\varphi^{*}=\left\{\varphi_{m}^{*}\right\}_{m=1}^{\infty}$. 
3.2. Let the family $\varphi$ satisfies the conditions of Theorem 1.2. In the first section we defined for each $f \in A_{\varphi}$ and $h \in \mathbb{Z}^{n}$ a sequence $f_{h}$ by the rule: $f_{h}(\alpha)=$ $f(\alpha+h), \alpha \in \mathbb{Z}^{n}$. Using the condition $\left.\mathrm{i}_{2}\right)$ on $\varphi$ for each $m \in \mathbb{N}$ we can find numbers $k \in \mathbb{N}$ and $d>0$ such that $p_{m}\left(f_{h}\right) \leqslant d p_{k}(f)$ for all $f \in A_{\varphi}$. Thus, for each $h \in \mathbb{Z}^{n}$ the linear operator $S_{h}: f \in A_{\varphi} \rightarrow f_{h}$ acts from $A_{\varphi}$ to $A_{\varphi}$ and is continuous. So if $H$ is a subset of $\mathbb{Z}^{n}$ consisting of finite number of elements and for $h \in H \quad \gamma_{h} \in \mathbb{C}$ then an operator $M: A_{\varphi} \rightarrow A_{\varphi}$ acting by the rule

is linear and continuous.

$$
M(f)=\sum_{h \in H} \gamma_{h} S_{h}(f), \quad f \in A_{\varphi}
$$

Let $g(z):=\sum_{h \in H} \gamma_{h} e^{-i\langle h, z\rangle} \quad\left(z \in \mathbb{C}^{n}\right)$. The function $g$ is usually called a characteristic function of the operator $M$.

3.2.1. In the proof of Theorems 1.2 and 1.3 the following lemma by L. Ehrenpreis and B. Malgrange (see, for example, Lemma A.1 in [3]) is used.

Lemma 3.3. Let $P$ be a polynomial of degree $m$. Then there exists a constant $C>0$ such that for every $r>0, z \in \mathbb{C}^{n}$ and every function $f$ which is defined for all $z^{\prime} \in \mathbb{C}^{n}$ with $\left\|z^{\prime}-z\right\|<r$ and is such that $\frac{f}{P}$ is holomorphic there, we have

$$
\left|\frac{f(z)}{p(z)}\right| \leqslant C r^{-m} \sup _{z^{\prime} \in B(z, r)}\left|f\left(z^{\prime}\right)\right| .
$$

Proof of Theorem 1.2. We have to prove that the operator $M$ is surjective on $A_{\varphi}$. First let us show that the image $i m M$ of the operator $M$ is dense in $A_{\varphi}$. Let $N_{g}=\left\{z \in \mathbb{C}^{n}: g(z)=0\right\}$. For $z \in \mathbb{C}^{n} \backslash N_{g}$ consider the equation $M(f)=f_{z}$. It has a solution $f=\frac{f_{z}}{g(z)}$ belonging to $A_{\varphi}$. From this and completeness of the system $\left\{f_{z}\right\}_{z \in \mathbb{C}^{n} \backslash N_{g}}$ we conclude that $i m M$ is dense in $A_{\varphi}$.

Let us show now that the image of the operator $M$ is closed in $A_{\varphi}$. It is known that closedness of image of $M$ in $A_{\varphi}$ is equivalent to closedness of the image of the adjoint operator $M^{*}$ in $A_{\varphi}^{*}$ (see [2], Theorem 8.6.13).

Consider an operator $\widehat{M}^{*}$ on $P_{\varphi^{\star}}$ acting by the rule:

$$
\widehat{M}^{*}(F)=\mathcal{F}\left(M^{*}\left(\mathcal{F}^{-1}(F)\right)\right), \quad F \in P_{\varphi^{\star}} .
$$

Obviously, $\widehat{M}^{*}$ is a linear continuous operator on $P_{\varphi^{\star}}$. Taking into account Theorem 1.1 we see that closedness of the image of the operator $M$ in $A_{\varphi}$ is equivalent to closedness of the image $i m \widehat{M}^{*}$ of the operator $\widehat{M}^{*}$ in $P_{\varphi^{\star}}$. Note that for each $F \in P_{\varphi^{\star}}$ and $z \in \mathbb{C}^{n}$

$$
\begin{aligned}
\widehat{M}^{*}(F)(z) & =\mathcal{F}^{-1}(F)\left(M\left(f_{z}\right)\right) \\
& =\mathcal{F}^{-1}(F)\left(\sum_{h \in H} \gamma_{h} S_{h}\left(f_{z}\right)\right)=\mathcal{F}^{-1}(F)\left(\sum_{h \in H} \gamma_{h}\left(e^{-i\langle\alpha+h, z\rangle}\right)_{\alpha \in \mathbb{Z}^{n}}\right) \\
& =\mathcal{F}^{-1}(F)\left(f_{z}\right) \sum_{h \in H} \gamma_{h} e^{-i\langle h, z\rangle}=F(z) g(z) .
\end{aligned}
$$

Thus, for each $F \in P_{\varphi^{\star}}$ we have $\widehat{M}^{*}(F)(z)=F(z) g(z), z \in \mathbb{C}^{n}$. 
By Theorem 1 in [7] $i m \widehat{M}^{*}$ is closed in $P_{\varphi^{*}}$ iff $i m \widehat{M}^{*} \cap P\left(\varphi_{m}^{\star}\right)$ is closed in $P\left(\varphi_{m}^{\star}\right)$ for each $m \in \mathbb{N}$. So let $m \in \mathbb{N}$ be arbitrary and $F$ belongs to the closure of $i m \widehat{M}^{*} \cap P\left(\varphi_{m}^{\star}\right)$ in $P\left(\varphi_{m}^{\star}\right)$. Then there exists a sequence $\left(F_{k}\right)_{k=1}^{\infty}$ of functions $F_{k} \in i m \widehat{M}^{*} \cap P\left(\varphi_{m}^{\star}\right)$ converging to $F$ in $P_{\varphi_{m}^{\star}}$. In particular, $F_{k} \rightarrow F$ uniformly on compact subsets of $\mathbb{C}^{n}$ as $k \rightarrow \infty$. So it is clear that the function $\psi(z)=\frac{F(z)}{g(z)}$ is holomorphic on $\mathbb{C}^{n}$. Obviously, $\psi(z+2 \pi l)=\psi(z)$ for all $z \in \mathbb{C}^{n}$ and $l \in \mathbb{Z}^{n}$. The functions $F$ and $\psi$ can be represented by the series

$$
\begin{array}{ll}
F(z)=\sum_{\alpha \in \mathbb{Z}^{n}} c_{\alpha} e^{-i\langle z, \alpha\rangle}, & z \in \mathbb{C}^{n}, \\
\psi(z)=\sum_{\alpha \in \mathbb{Z}^{n}} b_{\alpha} e^{-i\langle z, \alpha\rangle}, &
\end{array}
$$

As we know (see Lemma 2.1) the first series converges to $F$ in $P\left(\varphi_{m+k}^{\star}\right)$ for some $k \in \mathbb{N}$. The second series uniformly converges to $\psi$ on compact subsets of $\mathbb{C}^{n}$. Let us show that $\psi \in P_{\varphi^{\star}}$. Obviously, the functions $F_{0}(\zeta)=\sum_{\alpha \in \mathbb{Z}^{n}} c_{\alpha} \zeta^{\alpha}, \psi_{0}(\zeta)=$ $\sum_{\alpha \in \mathbb{Z}^{n}} b_{\alpha} \zeta^{\alpha}, g_{0}(\zeta)=\sum_{h \in H} \gamma_{h} \zeta^{h}$ are holomorphic in $(\mathbb{C} \backslash\{0\})^{n}$ and

$$
\psi_{0}(\zeta)=\frac{F_{0}(\zeta)}{g_{0}(\zeta)}, \quad \zeta \in(\mathbb{C} \backslash\{0\})^{n}
$$

Choose $N=\left(N_{1}, \ldots, N_{n}\right) \in \mathbb{Z}_{+}^{n}$ so that $P(\zeta)=g_{0}(\zeta) \zeta_{1}^{N_{1}} \cdots \zeta_{n}^{N_{n}}$ is a polynomial. Then

$$
\psi_{0}(\zeta)=\frac{F_{0}(\zeta) \zeta^{N}}{P(\zeta)}, \quad \zeta \in(\mathbb{C} \backslash\{0\})^{n} .
$$

Let us estimate $\left|\psi_{0}(\zeta)\right|$ from above at points $\zeta=\left(\zeta_{1}, \ldots, \zeta_{n}\right) \in(\mathbb{C} \backslash\{0\})^{n}$. Let

$$
\begin{aligned}
a_{m}(\zeta) & =\left(1+\left\|\left(\ln \left|\zeta_{1}\right|, \ldots, \ln \left|\zeta_{n}\right|\right)\right\|\right)^{\frac{1}{1-\mu_{m}}}, \\
r(\zeta) & =\min \left(1,\left(1-\exp \left(-\frac{a_{m}(\zeta)}{\sqrt{n}}\right)\right) \min _{1 \leqslant j \leqslant n}\left|\zeta_{j}\right|\right) .
\end{aligned}
$$

By Lemma 3.3 there exists a constant $C>0$ such that for all $\zeta \in(\mathbb{C} \backslash\{0\})^{n}$

$$
\begin{aligned}
\left|\psi_{0}(\zeta)\right| & \leqslant C(r(\zeta))^{-k} \sup _{w \in B(\zeta, r(\zeta))}\left(\left|F_{0}(w)\right|\|w\|^{|N|}\right) \\
& \leqslant C(r(\zeta))^{-k}(1+\|\zeta\|)^{|N|} \sup _{w \in B(\zeta, r(\zeta))}\left|F_{0}(w)\right| .
\end{aligned}
$$

Since for $\zeta=\left(\zeta_{1}, \ldots, \zeta_{n}\right) \in(\mathbb{C} \backslash\{0\})^{n}$

$$
\left|F_{0}(\zeta)\right| \leqslant\|F\|_{m} e^{\varphi_{m}^{\star}\left(\ln \left|\zeta_{1}\right|, \ldots, \ln \left|\zeta_{n}\right|\right)},
$$

then

$$
\left|\psi_{0}(\zeta)\right| \leqslant C(r(\zeta))^{-k}(1+\|\zeta\|)^{|N|} \exp \left(\sup _{w \in B(\zeta, r(\zeta))} \varphi_{m}^{\star}\left(\ln \left|w_{1}\right|, \ldots, \ln \left|w_{n}\right|\right)\right) .
$$


For points $w=\left(w_{1}, \ldots, w_{n}\right) \in B(\zeta, r(\zeta))$ we have

$$
\exp \left(\left(-\frac{a_{m}(\zeta)}{\sqrt{n}}\right)\left|\zeta_{j}\right| \leqslant\left|w_{j}\right| \leqslant \exp \left(\frac{a_{m}(\zeta)}{\sqrt{n}}\right)\left|\zeta_{j}\right|(j=1, \ldots, n) .\right.
$$

From this we get for $w \in B(\zeta, r(\zeta))$

$$
|\ln | w_{j}|-\ln | \zeta_{j}|| \leqslant \frac{a_{m}(\zeta)}{\sqrt{n}}, j=1, \ldots, n .
$$

Thus, for points $w=\left(w_{1}, \ldots, w_{n}\right) \in B(\zeta, r(\zeta))$

$$
\left\|\left(\ln \left|w_{1}\right|, \ldots, \ln \left|w_{n}\right|\right)-\left(\ln \left|\zeta_{1}\right|, \ldots, \ln \left|\zeta_{n}\right|\right)\right\| \leqslant\left(1+\left\|\left(\ln \left|\zeta_{1}\right|, \ldots, \ln \left|\zeta_{n}\right|\right)\right\|\right)^{\frac{1}{1-\mu_{m}}}
$$

Now using the inequality (7) we have

$$
\sup _{w \in B(\zeta, r(\zeta))} \varphi_{m}^{\star}\left(\ln \left|w_{1}\right|, \ldots, \ln \left|w_{n}\right|\right) \leqslant \varphi_{m}^{\star}\left(\ln \left|\zeta_{1}\right|, \ldots, \ln \left|\zeta_{n}\right|\right)+K_{m}
$$

From this and (9) we get

$$
\left|\psi_{0}(\zeta)\right| \leqslant C e^{K_{m}}(r(\zeta))^{-k}(1+\|\zeta\|)^{|N|} e^{\varphi_{m}^{\star}\left(\ln \left|\zeta_{1}\right|, \ldots, \ln \left|\zeta_{n}\right|\right)}
$$

for all $\zeta=\left(\zeta_{1}, \ldots, \zeta_{n}\right) \in(\mathbb{C} \backslash\{0\})^{n}$. Taking into account that for $z=\left(z_{1}, \ldots, z_{n}\right) \in$ $\mathbb{C}^{n}$ and $y=\operatorname{Imz}$

$$
a_{m}\left(e^{-i z_{1}}, \ldots, e^{-i z_{n}}\right)=(1+\|y\|)^{\frac{1}{1-\mu_{m}}}
$$

it is easy to show that $z \in \mathbb{C}^{n}$

$$
|\psi(z)| \leqslant C e^{K_{m}}\left(1+\frac{1}{\left(1-e^{-\frac{1}{\sqrt{n}}}\right) e^{-\|y\|}}\right)^{k}\left(1+\left\|\left(e^{\operatorname{Im} z_{1}}, \ldots, e^{\operatorname{Im} z_{n}}\right)\right\|\right)^{|N|} e^{\varphi_{m}^{\star}(\operatorname{Im} z)}
$$

From this we have

$$
|\psi(z)| \leqslant C e^{K_{m}}\left(\frac{2}{1-e^{-\frac{1}{\sqrt{n}}}}\right)^{k} e^{(|N|+k)\|\operatorname{Imz}\|}(1+n)^{|N|} e^{\varphi_{m}^{\star}(\operatorname{Im} z)} .
$$

Using the inequality (8) we obtain

$$
|\psi(z)| \leqslant K e^{\varphi_{m+k+|N|}^{\star}(\operatorname{Imz})}, \quad z \in \mathbb{C}^{n},
$$

where $K$ is some constant depending on $k, m,|N|, n$. Hence, $\psi \in P\left(\varphi_{m+k+|N|}^{\star}\right)$. Thus, $\psi \in P_{\varphi^{\star}}$. This means that $F \in i m \widehat{M}^{*}$. Hence, $i m \widehat{M}^{*} \cap P\left(\varphi_{m}^{\star}\right)$ is closed in $P\left(\varphi_{m}^{\star}\right)$ for each $m \in \mathbb{N}$. Thus, the image of the operator $\widehat{M}^{*}$ is closed in $P_{\varphi^{\star}}$. Therefore, the image of the operator $M$ is closed in $A_{\varphi}$.

Thus, the image of the operator $M$ is dense and closed in $A_{\varphi}$. Therefore, $i m M=A_{\varphi}$. The proof is complete. 
3.2.2. Consider the equation $M(f)=\mathbf{0}$ in $A_{\varphi}$. Note that if $f$ belongs to the kernel $W$ of the operator $M$ then for each $\beta \in \mathbb{Z}^{n} \quad S_{\beta}(f) \in W$.

Recall that for $\mu \in \mathbb{Z}_{+}^{n}$ and $\zeta \in \mathbb{C}^{n}$ we defined the sequence $E_{\mu, \zeta}$ by the rule: $\alpha \in \mathbb{Z}^{n} \rightarrow \alpha^{\mu} e^{-i\langle\alpha, \zeta\rangle}$.

Lemma 3.4. The sequence $E_{\mu, \zeta}$ is in $W$ iff $\left(D^{\beta} g\right)(\zeta)=0$ for all $\beta \in \mathbb{Z}_{+}^{n}$ such that $\beta \leqslant \mu$.

Proof. Consider more complicated case $n \geqslant 2$. Let $\mu \in \mathbb{Z}_{+}^{n}$ and $\zeta \in \mathbb{C}^{n}$ be such that $\left(D^{\beta} g\right)(\zeta)=0$ for all $\beta \in \mathbb{Z}_{+}^{n}$ such that $\beta \leqslant \mu$. Then for each $\alpha \in \mathbb{Z}^{n}$

$$
\begin{aligned}
M\left(E_{\mu, \zeta}\right)(\alpha) & =\sum_{h \in H} \gamma_{h}(\alpha+h)^{\mu} e^{-i\langle\alpha+h, \zeta\rangle} \\
& =e^{-i\langle\alpha, \zeta\rangle} \sum_{h \in H} \gamma_{h}(\alpha+h)^{\mu} e^{-i\langle h, \zeta\rangle} \\
& =e^{-i\langle\alpha, \zeta\rangle} \sum_{\beta \in \mathbb{Z}_{+}^{n}: \beta \leqslant \mu} C_{\mu}^{\beta} \alpha^{\mu-\beta} \sum_{h \in H} \gamma_{h} h^{\beta} e^{-i\langle h, \zeta\rangle} \\
& =e^{-i\langle\alpha, \zeta\rangle} \sum_{\beta \in \mathbb{Z}_{+}^{n}: \beta \leqslant \mu} C_{\mu}^{\beta} \alpha^{\mu-\beta} i^{|\beta|}\left(D^{\beta} g\right)(\zeta)=0 .
\end{aligned}
$$

Thus, $E_{\mu, \zeta} \in W$.

Now let for some $\mu \in \mathbb{Z}_{+}^{n}$ and $\zeta \in \mathbb{C}^{n} \quad E_{\mu, \zeta} \in W$. Then for each $\alpha \in \mathbb{Z}^{n}$

$$
\sum_{h \in H} \gamma_{h}(\alpha+h)^{\mu} e^{-i\langle\alpha+h, \zeta\rangle}=0
$$

Hence, for each $\alpha \in \mathbb{Z}^{n}$

$$
\sum_{h \in H} \gamma_{h}(\alpha+h)^{\mu} e^{-i\langle h, \zeta\rangle}=0
$$

In particular, $\sum_{h \in H} \gamma_{h} h^{\mu} e^{-i\langle h, \zeta\rangle}=0$. This means that $\left(D^{\mu} g\right)(\zeta)=0$. Further, let $\nu=\left(\nu_{1}, \ldots, \nu_{n}\right) \in \mathbb{Z}_{+}^{n}$ be such that $\left(D^{\beta} g\right)(\zeta)=0$ for all $\beta \in \mathbb{Z}_{+}^{n}$ such that $\nu \leqslant \beta \leqslant \mu$. If $\nu=(0, \ldots, 0)$ then Lemma holds. If $\nu \neq(0, \ldots, 0)$ then there exists $j \in\{1, \ldots, n\}$ such that $\nu_{j} \geqslant 1$. For simplicity suppose $j=1$ and show that $\left(D^{\left(\beta_{1}, \nu_{2}, \ldots, \nu_{n}\right)} g\right)(\zeta)=0$ for all $\beta_{1}=0, \ldots, \nu_{1}-1$. For each $\alpha \in \mathbb{Z}^{n}$

$$
\begin{aligned}
\sum_{h \in H} \gamma_{h}(\alpha+h)^{\mu} e^{-i\langle h, \zeta\rangle} & =\sum_{h \in H} \gamma_{h} \sum_{(0, \ldots, 0) \leqslant \beta \leqslant \mu} C_{\mu}^{\beta} \alpha^{\mu-\beta} h^{\beta} e^{-i\langle h, \zeta\rangle} \\
& =\sum_{(0, \ldots, 0) \leqslant \beta \leqslant \mu} C_{\mu}^{\beta} \alpha^{\mu-\beta} \sum_{h \in H} \gamma_{h} h^{\beta} e^{-i\langle h, \zeta\rangle} \\
& =\sum_{(0, \ldots, 0) \leqslant \beta \leqslant \mu} C_{\mu}^{\beta} \alpha^{\mu-\beta} i^{|\beta|}\left(D^{\beta} g\right)(\zeta) \\
& =\sum_{(0, \ldots, 0) \leqslant \beta \leqslant \nu, \beta \neq \nu} C_{\mu}^{\beta} \alpha^{\mu-\beta} i^{|\beta|}\left(D^{\beta} g\right)(\zeta) .
\end{aligned}
$$


In view of $(10)$

$$
\sum_{(0, \ldots, 0) \leqslant \beta \leqslant \nu, \beta \neq \nu} C_{\mu}^{\beta} \alpha^{\mu-\beta} i^{|\beta|}\left(D^{\beta} g\right)(\zeta)=0, \quad \alpha \in \mathbb{Z}^{n} .
$$

From this we have for all $\alpha \in \mathbb{Z}^{n}$

$$
\sum_{\beta_{1}=0}^{\nu_{1}} C_{\mu_{1}}^{\beta_{1}} \alpha_{1}^{\nu_{1}-\beta_{1}} i^{\beta_{1}} \sum_{(0, \ldots, 0) \leqslant \beta^{\prime} \leqslant \nu^{\prime}} \alpha^{\prime \nu^{\prime}-\beta^{\prime}} i^{\left|\beta^{\prime}\right|}\left(D^{\beta} g\right)(\zeta)=0 .
$$

Putting here $\alpha_{2}=\cdots=\alpha_{n}=0$ we get for all $\alpha_{1} \in \mathbb{Z}$

$$
\sum_{\beta_{1}=0}^{\nu_{1}-1} C_{\mu_{1}}^{\beta_{1}} \alpha_{1}^{\nu_{1}-\beta_{1}} i^{\beta_{1}}\left(D^{\left(\beta_{1}, \nu_{2}, \ldots, \nu_{n}\right)} g\right)(\zeta)=0 .
$$

From this it follows that $\left(D^{\left(\beta_{1}, \nu_{2}, \ldots, \nu_{n}\right)} g\right)(\zeta)=0$ for all $\beta_{1}=0, \ldots, \nu_{1}-1$. Obviously, applying these arguments so on we will obtain that $\left(D^{\beta} g\right)(\zeta)=0$ for all $\beta \in \mathbb{Z}_{+}^{n}$ such that $\beta \leqslant \mu$.

The proof of Lemma is complete.

Let $\mathcal{A}=\left\{(\mu, \zeta) \in \mathbb{Z}_{+}^{n} \times \mathbb{C}^{n}: E_{\mu, \zeta} \in W\right\}$. Recall that $\mathcal{E}$ is a set of all solutions of the form $E_{\mu, \zeta}$ of the equation $\sum_{h \in H} \gamma_{h} f_{h}=\mathbf{0}$.

Proof of Theorem 1.3. Let $S$ be a linear continuous functional on $A_{\varphi}$ such that $S(f)=0$ for each $f \in \mathcal{E}$. If we will show that $S(f)=0$ for each $f \in W$ then a closure of a linear envelope of $\mathcal{E}$ will coincide with $W$. Using Lemma 3.4 and taking account that for all $(\mu, \zeta) \in \mathcal{A}\left(D^{\beta} \hat{S}\right)(\zeta)=0$ for $\beta \in \mathbb{Z}_{+}^{n}$ such that $\beta \leqslant \mu$ it is easy to check that the function $\psi:=\frac{\hat{S}}{g}$ is entire. From the proof of Theorem 1.2 it follows that $\psi \in P_{\varphi^{\star}}$. Hence, by Theorem 1.1 there exists a functional $\Psi \in A_{\varphi}^{\prime}$ such that $\hat{\Psi}=\psi$. Obviously, the functional $\Psi \circ M$ is in $A_{\varphi}^{\prime}$ too. And for each $z \in \mathbb{C}^{n}$ we have $\mathcal{F}(\Psi \circ M)(z)=\Psi\left(M\left(f_{z}\right)\right)=\Psi\left(g(z) f_{z}\right)=\psi(z) g(z)=\hat{S}(z)$. By theorem $1 \Psi \circ M=S$. Now for each $f \in W$ we have

$$
S(f)=(\Psi \circ M)(f)=\Psi(M(f))=0 .
$$

Thus, a linear envelope of $\mathcal{E}$ is dense in $W$.

\section{References}

[1] A.A. Borichev, Convolution equations in spaces of sequences of exponential growth, Journal of Soviet Mathematics 42 (1988), no. 2, 1614-1620; translated from Zapiski Nauchnykh Seminarov Leningradskogo Otdeleniya Matematicheskogo Instituta im. V.A. Steklova AN SSSP, 149 (1986), 107-115.

[2] R.E. Edwards, Functional Analysis, Holt, Pineart and Winston, 1965. 
[3] S. Hansen, On the "Fundamental Principle" of L. Ehrenpreis, Partial differential equations, Banach center publications, Warsaw, PWN-Polish Scientific Publishers, 10 (1983), 185-203.

[4] C. Rényi, On periodic entire functions, Acta Math. Acad. Sci. Hungar. 7 (1956), 145-149.

[5] A.P. Robertson, W. Robertson, Topological Vector Spaces, Cambridge Univ. Press, Cambridge, 1980.

[6] L.A. Rubel, B.A. Taylor, Weighted polynomial approximation on integers, Archiv der Mathematik 19 (1968), no. 5, 511-515.

[7] J. Sebastiào e Silva, Su certe classi di spazi localmente convessi importanti per le applicazioni, Rend. Mat. e Appl. 14 (1955), 388-410.

[8] V.V. Zharinov, Compact families of locally convex topological vector spaces, Fréchet-Schwartz and dual Fréchet-Schwartz spaces, Uspekhi Mat. Nauk 34 (1979), no. 4, 97-131.

Addresses: Nadir V. Ibadov: Vice-Rector for Scientific and Technical Affairs, Ganja State University, 187, Heydar Aliyev Avenue, Ganja, AZ-2000, Azerbaijan;

Il'dar Kh. Musin: Institute of Mathematics, Russian Academy of Sciences, 112 Chernyshevsky str., 450008, Ufa, Russia.

E-mail: nadir.ibadov@gmail.com, musin@matem.anrb.ru

Received: 9 November 2012; revised: 18 March 2013 